\title{
Is it possible to block the cancer cells in circulating blood by extracorporeal circulation? Implications of the immune system and other factors (review)
}

\author{
Sante Basso Ricci $^{1}$, Francesco Garbagnati ${ }^{{ }^{*}}$ \\ ${ }^{1}$ Department of Radiotherapy, Fondazione IRCCS Istituto Nazionale Tumori, Milan, Italy \\ ${ }^{2}$ Radiology Department, Fondazione IRCCS Istituto Nazionale Tumori, Milan, Italy; \\ *Corresponding Author: francesco.garbagnati@istitutotumori.mi.it
}

Received 14 October 2013; revised 14 November 2013; accepted 21 November 2013

Copyright (c) 2013 Sante Basso Ricci, Francesco Garbagnati. This is an open access article distributed under the Creative Commons Attribution License, which permits unrestricted use, distribution, and reproduction in any medium, provided the original work is properly cited. In accordance of the Creative Commons Attribution License all Copyrights (c) 2013 are reserved for SCIRP and the owner of the intellectual property Sante Basso Ricci, Francesco Garbagnati. All Copyright (C 2013 are guarded by law and by SCIRP as a guardian.

\begin{abstract}
The presence of cancer cells in circulating blood and the possibility of colonization in the tissues of the body worsen in a determinant way the prognosis of the disease. Nevertheless, there are factors in the body that can lead to a prognostic improvement, even in such conditions. In addition to the immune system, other favorable factors can act at the level of the microenvironment of the tumor so much that cases of spontaneous total regression, not only of primary tumors but also metastases, have been reported in the literature. Since it was recently reported that patients with renal tumors and in permanent hemodialysis present at post mortem examination, a metastatic spread much less extensive than observed in patients deceased for renal tumors but not on hemodialysis. The authors maintain it likely that the dialytic membrane concurs to block at least in part the cancer cells circulating in the blood. The possibility to block cancer cells can be extended in addition to the dialytic membrane also to other types of filters inserted in the extracorporeal circulation. The block of the cancer cells thus is obtained and that occurs in regional lymph nodes and in the microenvironment of the tumor causing a relative increase in the elements of the immune system compared to the number of cancer cells, which could determine clearly positive therapeutic results also in cases with advanced metastatic spread.
\end{abstract}

Keywords: Block of Cancer Cells; Extracorporeal Circulation; Filters; Immune System

\section{BACKGROUND}

Metastatic spread of malignancies in the circulating blood leads to a marked prognostic worsening, which in most cases is irremediable. In fact, the presence of cancer cells in the blood leads to the possibility of colonization of the neoplasm in all the tissues of the organism. Nevertheless, the organism's defense remains active and can oppose the neoplastic disease. However, cases of spontaneous regression not only of the primary tumor but also of metastases have been reported [1,2], so it is possible that situations favorable for the prognosis may be present also in advanced stages of spread of the neoplastic disease [3].

In fact, the immune system remains active, also in such an advanced state of diffusion, even though its action is linked, in the case of malignant neoplasms, prevalently to natural immunity. Moreover, other factors also remain active, which are mentioned further on. Unfortunately, all these factors may be absent or present in an insufficient quantity. Still, in cases in which they are present, a summation of effects is possible, which is useful for clinical evolution of the disease, associating these factors also with the therapeutic treatments. If to such a possibility one adds that offered by eventual filters which, inserted by techniques of extracorporeal circulation of the blood, can block even though partially the cancer cells circulating in the blood, it is permissible to hope to efficiently block metastatic diffusion via the blood. 


\section{FACTORS THAT ARE EFFICIENT TO IMPOSE SUCH A BLOCK}

For the problems relative to the block of the cancer cells and therefore to the lack of their development, the following factors should be considered.

1) The presence of factors in the microenvironment of the tumor that block the neoplastic cells in the tissues of the organism. The absence of factors that allow the development of neoplastic cells.

Among the factors that block the development of the neoplasm at the level of the microenvironment of the tumor, there is mainly the eventual presence of inhibitors of metalloproteinases and of angiogenesis, since metalloproteinases initiate neoplastic infiltration and angiogenesis is indispensable for development of the tumor. The eventual presence of such factors and the absence of other factors, such as epithelial cadherin proteins [4], elements of the large integrin family [5], and adenosine [6], impede development of the neoplasm.

All the factors that inhibit the development of the neoplasm can effectively block in the tissues eventual metastatic cells that arrive via the circulating blood as well as infiltration of the primary tumor in surrounding tissues. Unfortunately, such conditions are realized together only in a small number of cases and, even when combined with other favorable conditions, may seldom be of clinical importance.

2) Factors linked to the immune system that tend to eliminate the neoplastic cells in the circulating blood as well as in tissues of the organism.

The component of the immune system that opposes the neoplastic disease is represented mainly by natural immunity, even if in some cases also by the intervention of adaptive immunity. Among the other elements of the immune system most active against neoplastic disease, leukocytes, including $\mathrm{T}$ lymphocytes and natural killer (NK) cells, are active at the level of the primary tumor as well as at the level of the neoplastic cells circulating in the blood. Leukocytes can infiltrate the primary tumor or metastases and act with a cytolytic action on the neoplastic cells. NK cells and T lymphocytes act electively on the cancer cells circulating in the blood.

It should also be noted that during the process of carcinogenesis and the development of primary tumors, as well as during metastatic spread in the blood and finally during the development of metastases, the damage effected by various elements of the immune system is accumulated to overcome the resistance threshold of the cancer cells and kill them or to create a condition of preapoptosis. The summation of cellular damage is possible because the damage is always of the same type, i.e., cytolytic.

3) Lymph node metastasis
Metastatic cells can reach the lymph nodes via the lymphatic vessels. These cells are first blocked in the lymph nodes and only after metastatic colonization occurs, causing extranodal spread. At the level of lymph nodes, the immune system is very active, and it cannot be excluded that in many cases with microscopic diffusion the neoplastic cells do not colonize. In any case, such metastases could represent a stimulus for the immune system. In fact, cases of spontaneous regression of already colonized lymph node metastases have been reported [7]. It should be noted with regard to our argument that lymph nodes also concur in a certain number of cases to block neoplastic cells that detach from the primary tumor.

4) Possibilities of techniques of extracorporeal circulation of the blood to block neoplastic cells

From the comparison of two series of patients deceased for renal carcinoma, one of patients in permanent hemodialysis until death and the other of patients not subjected to hemodialysis, it was deduced, after post mortem examination, that the number of metastases diffused in the organism was markedly higher in the patients not subjected to hemodialysis. The results appeared referable to the presence of the dialytic membrane and to the possible block of cancer cells circulating in the blood [8-10].

It is possible to think that the therapeutic result, i.e., the block of neoplastic cells, can be obtained with at least two types of filters inserted in the extracorporeal circulation of blood. One type of filter is represented by the dialytic membrane, thus characterized by pores of molecular dimension that exclude the corpuscular component of the blood, and another type of filter that allows the transit of blood cells, thereby selecting abnormal cells.

It should be remembered that for the existence of the process of adhesion, indispensable because biological processes of great importance may be present, multicellular complexes tend to form in the blood with sizes markedly increased with respect to the single cells and with irregular morphology [11]. In particular, multicellular complexes made up of NK cells and cancer cells circulating in the blood should be considered. It is possible to find electron-dense regions in relation to certain areas of the cell membrane that are much closer to the underlying substrate [12,13]. These electron charges are conditioned by alterations of the underlyng substrate [14] and thus by factors that alter the substrate, as in our case of cytolytic alterations in the context of cancer cells or also consequent to therapeutic treatments (such as chemotherapy). The electron charges can react with the forces of van der Waals of the non-biological structures of filters and thus favor the block of cancer cells. In this way, particular conditions that can be explained by biophysi- 
cal studies are created. In any case, the block of metastatic cells can occur on the surface of molecular pores of the dialytic membrane or in the mesh of other filters.

Moreover, to complete the description of the conditions that favor the block of cancer cells at the level of filters, and that is the action of attractive and repulsive forces, it should be added that such forces are not necessarily opposite in realizing the block at the level of filters of extracorporeal circulation. In fact, whereas the propulsive force, in particular that represented by the blood stream, pushes the cancer cells in the filters (those types of filters that allow the passage of cells), the repulsive force stops the passage thus determining a block of the cells in the filters. It should be remembered that these filters are acceptable for therapeutic purposes on the condition that their biocompatibility is ascertained. Hemolysis and deterioration of proteins and embolism might be the main hazards of these filters.

For the filters of the dialytic membrane, particular phenomena should be remembered, like an increase in some cytokines (mainly IL 18 and TNF-alpha) and an increase in complement, especially in the presence of phlogistic processes [15]. In this regard, we should remember that the cuprophane membrane is associated, in the presence of complement, to abundant production of cytokines, whereas other types of membranes have not been found to be related to alteration of circulating cytokine levels [16,17]. Again in relation to the dialytic membrane, it should be kept in mind that hemodialysis with a complement-activating membrane induces neutropenia and rebound leukocytosis, which is associated with increased cell adhesion [18].

The adhesion process is cytokine inducible and mediated by the interaction of four classes of adhesion molecules: selectins, carbohydrate-containing selectin ligands, integrins and immunoglobulin (Ig)-like molecules [19, 20]. Such molecules can contribute to the formation of multicellular complexes that include the neoplastic cells and therefore increase the probability that such complexes are blocked by the dialytic membrane in patients on chronic hemodialysis. This fact could explain the relatively small number of metastases found post mortem in patients on chronic hemodialysis and deceased for renal tumors. In the case of patients on hemodialysis, the varying influence of uremia on the adhesion process should be considered.

5) The block of cancer cells determines a relative increase in cells of the immune system

The block of cancer cells in the tissues, in lymph nodes and in filters of extracorporeal circulation determines a relative increase in the cells of the immune system circulating in the blood, i.e., an increased value of the ratio between cells of the immune system and cancer cells circulating in the blood, with positive clinical re- sults. Instead, a generic stimulus, like that generally obtained with medical treatment, would incur in the risk of a feedback mechanism that frequently involves the immune system, and clinical results might be negative. For this reason, it is justified to hope that the block of cancer cells, effected by filters of extracorporeal circulation, might offer better clinical results.

It should be added that the relative increase in elements of the immune system is increased further on, in addition to the block of cancer cells, by the presence of eventual filters, even by the block of such cells in the lymph nodes and in other tissues for the eventual presence of inhibitors of metalloproteinases and angiogenesis, as well as for the absence of neoplastic growth factors, as mentioned before. So that, even if all these possibilities of a block are non constant and exclusively local, they could improve the prognosis of the disease for the effect of a relative increase in the elements of the immune system.

The relative increase in the elements of the immune system may concur also to eliminate probable metastatic colonizations already in act in the tissues, because it allows a greater infiltration, particularly of $\mathrm{T}$ lymphocytes in the metastatic neoformations.

\section{CONCLUSIONS}

The application of techniques of extracorporeal circulation of the blood with particular filters and the block, even partial, of cancer cells circulating in the blood can lead to a prognostic improvement of the disease, especially if associated with other favorable conditions. Such conditions are the block of cancer cells in lymph nodes or in other tissues of the body, the presence of inhibitors of metalloproteinases and angiogenesis, the absence of factors indispensable to the development of the neoplasm, and finally the activity of the immune system. For the effect of such blocks, the value of the ratio between cells of the immune system and cancer cells increases. In particular, the number of elements of the immune system (NK cells and T lymphocytes) circulating in the blood increases comparatively to the number of cancer cells in the blood. Thus, the favorable effect of the filters is twofold: the direct block of the cancer cells and the relative increase in the cells of the immune system.

The relative and not absolute increase in the elements of the immune system thus avoids the risk of a feedback reaction, which could instead accompany the not sufficiently specific stimulation of the immune system with a therapeutic purpose. The immune system is important in the block of cancer cells because NK cells and T lymphocytes concur to form multicellular complexes that have greater possibilities to be blocked by filters compared to monocellular elements, at least as regards the 
non-molecular filters.

Since in most cases elements of the immune system are present in the multicellular complexes, one can think that the block of such complexes is useful, in addition to oncologic aims, also in the case of autoimmune diseases, because the block leads to the elimination of active autoimmune elements.

Filters used in the technique of extracorporeal circulation of the blood with the scope of blocking cancer cells can be of a type like the dialytic membrane with molecular pores. Obviously, the use of filters with molecular pores and for oncological purposes does not impose a complete hemodialytic treatment but requires technical modifications so that the hematic component filtered through the pores can be recuperated and the debilitating consequences of the dialytic treatment can be avoided. Other types of filters with wider holes allow the passage of cells and their selective effect in relation to the form and size of the neoplastic cells or of the multicellular complexes formed for the effect of the process of adhesion. Electron charges of the cell surface and van der Waals forces of the nonbiological component of the filters are important for the block of the cancer cells.

\section{REFERENCES}

[1] Thoroddsen, A., Geirsson, G., Agnarson, B.A. and Magnusson, K. (2002) Spontaneous regression of pleural metastases after nephrectomy for renal cell carcinoma. Scandinavian Journal of Urology and Nephrology, 5, 396-398. http://dx.doi.org/10.1080/003655902320783971

[2] De Weerd, J.H., Hawthorn, J. and Adson, M.A. (1977) Regression of renal cell hepatic metastases following removal of primary lesions. Journal of Urology, 117, 790792.

[3] Basso Ricci, S. and Cerchiari, U. (2010) Spontaneous regression of malignant tumors: importance of the immune system and other factors (review). Oncology Letters, 1, 941-945.

[4] Munshi, H.G. and Stack, M.S. (2006) Reciprocal interactions between adhesion receptor signaling and MMP regulation. Cancer Metastasis Review, 25, 45-56. http://dx.doi.org/10.1007/s10555-006-7888-7

[5] Hood, J.D. and Cheresh, D.A. (2002) Role of integrins in cell invasion and migration. National Review of Cancer, 2, 91-100. http://dx.doi.org/10.1038/nrc727

[6] Hoskin, D.H., Mader, J.S., Furlong, S.J., Conrad, D.M. and Blay, J. (2008) Inhibition of T cell and natural killer cell function by adenosine and its contribution to immune evasion by tumor cells (Review). International Journal of Oncology, 18, 527-535.

[7] Kurita, M., Hirano, K., Ebihara, S., Takushima, A., Hari, K., Fujino, T. and Fujioka, Y. (2007) Spontaneous regression of cervical lymph node metastasis in patients with nasopharyngeal squamous cell carcinoma of the tongue: Possible association between apoptosis and tumor regression. Journal of Clinical Oncology, 12, 448-454. http://dx.doi.org/10.1007/s10147-007-0711-9

[8] Basso Ricci, S. (2007) Dialysis membrane and metastatic cancer cells. Clinical Nephrology, 68, 354-356. http://dx.doi.org/10.5414/CNP68354

[9] Basso Ricci, S. and Cerchiari, U. (2009) Some relations among the dialysis membrane, metastatic cells and the immune system. Medical Hypotheses, 73, 328-331. http://dx.doi.org/10.1016/j.mehy.2009.03.043

[10] Basso Ricci, S. (2012) Therapeutic possibilities of techniques of extracorporeal blood circulation in oncology. Medical Hypotheses, 78, 1-3. http://dx.doi.org/10.1016/j.mehy.2011.09.026

[11] Witz, I.P. (2008) The selectin-selectin ligand axis in tumor progression. Cancer Metastasis Review, 27, 18-30. http://dx.doi.org/10.1007/s10555-007-9101-z

[12] Abercombe, M., Heasman, J. and Pegrum, S.M. (1975) The locomotion of fibroblasts in culture. Experimental Cell Research, 67, 359-367. http://dx.doi.org/10.1016/0014-4827(71)90420-4

[13] Abercombe, M. and Dunn, G.A. (1975) Adhesion fibroblasts to substratum during contact inhibition observed by interference reflection microscopy. Experimental Cell Research, 92, 57-62. http://dx.doi.org/10.1016/0014-4827(75)90636-9

[14] Lo, S.H. and Chen, L.B. (1994) Focal adhesion as a signal transduction organelle. Cancer Metastasis Review, 132, 9-24. http://dx.doi.org/10.1007/BF00690415

[15] Rousseau, Y., Haeffner-Cavaillon, N., Poignet, J.L., Meyrier, A. and Carreno, M.P. (2000) In vitro intracellular cytokine production by leucocytes during hemodialysis. Cytokine, 12, 506-517. http://dx.doi.org/10.1006/cyto.1999.0574

[16] Bingel, M., Lonnemann, G., Koch, K.M., Dinarello, A. and Sheldon, S. (1988) Plasma interleukin-1 activity during hemodialysis: The influence of dialysis membrane. Nephron, 50, 273-276. http://dx.doi.org/10.1159/000185186

[17] Canivet, E., Lavand, S., Wong, T., Guenounoue, M., Willemin, J.C., Potron, G. and Chanard, J. (1994) Cuprophane but not synthetic membrane induces increases in serum tumor necrosis factor alpha levels during hemodialysis. American Journal of Kidney Disease, 23, 41-46.

[18] Arnaut M.A., Kakim R.M. and Todd R.F. (1985) Increased expression of an adhesion-promoting surface glycoprotein in the granulocytopenia of hemodialysis. New England Journal of Medicine, 312, 457-462. http://dx.doi.org/10.1056/NEJM198502213120801

[19] Dal Canton, A. (1993) Adhesion molecules in renal disease. Kidney International, 48, 1087-1090.

[20] Mrowka, C., Heintz, B. and Siebert, H.G. (1999) Is dialysis membrane type responsible for increased circulating adhesion molecules during chronic hemodialysis? Clinical Nephrology, 52, 312-321. 